40th "Jaszowiec" International School and Conference on the Physics of Semiconductors, Krynica-Zdrój 2011

\title{
Terahertz Detection of Quantum Cascade Laser Emission by Plasma Waves in Field Effect Transistors
}

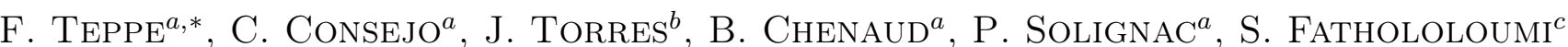
Z.R. Wasilewski ${ }^{c}$, M. Zholudev ${ }^{a, d}$, N. Dyakonova ${ }^{a}$, D. Coguillat $^{a}$, A. El Fatimy ${ }^{e}$ P. Buzatu ${ }^{a}$, C. Chaubet $^{a}$ And W. KnaP ${ }^{a}$

${ }^{a}$ L2C, UMR N $N^{\circ} 5221$ CNRS, Université Montpellier 2, GIS-TERALAB, 34095 Montpellier, France

${ }^{b}$ IES, UMR No 5214 CNRS, Université Montpellier 2, GIS-TERALAB, 34095 Montpellier, France

${ }^{c}$ Institute for Microstructural Sciences, National Research Council, Ottawa, Canada

${ }^{d}$ Institute for Physics of Microstructures, RAS, Nizhny Novgorod, Russia

${ }^{e}$ School of Physics and Astronomy, Cardiff University, Cardiff, United Kingdom

\begin{abstract}
We report on the resonant detection of a $3.1 \mathrm{THz}$ radiation produced by a quantum cascade laser using a $250 \mathrm{~nm}$ gate length GaAs/AlGaAs field effect transistor at liquid nitrogen temperature. We show that the physical mechanism of the detection is related to the plasma waves excited in the transistor channel. The detection is enhanced by increasing the drain current and driving the transistor into saturation regime. These results clearly show that plasma wave nanometer-size transistors can be used as detectors in all-solid-state terahertz systems where quantum cascade lasers act as sources.
\end{abstract}

PACS: 85.30.Tv, 07.57.Kp, 52.35.-g

\section{Introduction}

The quantum cascade laser (QCL) terahertz (THz) sources are well known to be one of most important sources in modern $\mathrm{THz}$ physics $[1,2]$. Nevertheless, most of the detectors in the $\mathrm{THz}$ frequency range are cumbersome and/or require cryogenic temperature operation. The development of $\mathrm{THz}$ systems suffers from the lack of compact and fast detectors that would allow constructing detector arrays/cameras for $\mathrm{THz}$ space and for time resolved imaging to investigate materials/devices/ structures in chemistry, biology, and medicine [3]. Consequently, a significant effort must be devoted to the study and development of solid-state tunable detectors of radiations above $1 \mathrm{~T}$ where many substances have fingerprint lines. Nonlinearities related to plasma wave excitations in two-dimensional electron gas (2DEG) in a nanometer size field effect transistor (FET) were proposed by Dyakonov and Shur as a new way to realize selective resonant and voltage tunable $\mathrm{THz}$ detectors [4]. In the framework of this theory, in absence of drain current, a FET subject to electromagnetic radiation can develop a constant drainsource voltage,

$$
\Delta U \propto \frac{\omega_{0}^{2}}{\left(\omega-\omega_{0}\right)^{2}+(1 / 2 \tau)^{2}},
$$

where $\tau=m^{*} \mu / e$ is the momentum relaxation time, $\mu$ is the electron mobility, $m^{*}$ is the electron effective mass and $e$ is the electronic charge. When the quality factor of

* corresponding author; e-mail: frederic.teppe@univ-montp2.fr the resonant cavity $\omega_{0} \tau \ll 1$, a strong damping of plasma oscillations occur and the FET response is a smooth function of the temperature as well as of the gate voltage. For $\omega_{0} \tau \gg 1$, the linewidth $1 / \tau$ is small and FET operates as a resonant detector. Resonant and non-resonant detection of $\mathrm{THz}$ radiation by two-dimensional plasma waves was demonstrated several times using a commercial GaAs/AlGaAs FET at cryogenic [5] and room temperature $[6-8]$ and using different laboratory $\mathrm{THz}$ sources such as backward wave oscillator (BWO), Gunn diodes, molecular lasers, etc...

In this letter, we present experimental evidences of the plasma wave resonant detection of $3.1 \mathrm{THz}$ quantum cascade laser radiation by GaAs FETs working at liquid nitrogen temperature and which can be inserted in a compact all-solid-state $\mathrm{THz}$ system.

\section{Experimental setup}

The experiments were performed on a $250 \mathrm{~nm}$ gate-length GaAs Fujitsu FET (FHX06X [9]). The threshold voltage, extracted from $I-V$ characteristics was around $U_{\text {th }}=-0.45 \mathrm{~V}$. The sample was mounted on a Dual In Line package, and was placed on a cold finger inside a liquid nitrogen cryostat behind a crystalline quartz window (Fig. 1). The temperature of the FET was approximately $90 \mathrm{~K}$.

The QCL used as a source in our experiments emits a radiation at $3.1 \mathrm{THz}$ and was placed in a closed-cycle cryostat. The repetition rate of the laser was $3 \mathrm{kHz}$; the width of the pulses was $3 \mu \mathrm{s}$. The pulsed $\mathrm{THz}$ radiation was collimated and focused onto the detector by using 


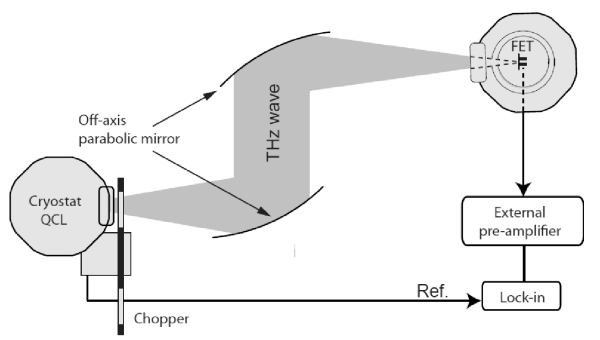

Fig. 1. Schematic of the experimental setup showing the two cryostats where there are placed the QCL and the plasma wave detector and their associated optics.

two parabolic off-axis mirrors. No special coupling antennas were used and the radiation was coupled to the device through the contact pads. The radiation intensity was modulated with a mechanical chopper at $40 \mathrm{~Hz}$. The device was tuned by a dc-gate voltage bias and the FET was biased in a common source configuration. The induced dc drain voltage, $\Delta U$ which appeared in response to the modulated $\mathrm{THz}$ radiation was superimposed on a controlled dc drain current $I_{\mathrm{ds}}$ driven through the device using a Keithley Source Meter 2410. The detector signal $\Delta U$ was measured by using a standard lock-in technique at different dc drain voltage $V_{\mathrm{d}}$ monitored by the drain current.

\section{Results and discussion}

Figure 2 reports the photoresponse plotted as a function of the gate voltage for different values of the applied drain voltage. The QCL's and FET's temperatures were $40 \mathrm{~K}$ and $90 \mathrm{~K}$, respectively. The signal exhibits a very well pronounced peak around $-0.2 \mathrm{~V}$. By increasing the drain voltage, the maximum of the curves increases from $20 \mu \mathrm{V}$ up to $60 \mu \mathrm{V}$. One has to notice that without applied current the signal was lower than the noise level and consequently not visible. Accordingly with Teppe et al. $[6,7]$ who have shown that the increase of the drain current up to the saturation regime leads to the increase of the non-resonant detection and its transformation to the resonant one at $300 \mathrm{~K}$, results of Fig. 2 can be explained by the changes of the electron drift velocity in the channel. Indeed, in $[6,7]$ the detection becomes resonant when the plasma wave attenuation is enough affected to lead to a quality factor $\omega_{0} \tau_{\text {eff }} \approx 1$. In the present experiments the quality factor is theoretically higher than unity even without current because of the high incident frequency and the high electron mobility at low temperature. With a minimal relaxation time of $0.39 \mathrm{ps}$, the quality factor is estimated around 7 at $3.1 \mathrm{THz}$. This value and the FET geometrical parameters have been used in the calculation of Fig. 3.

According to plasma wave detection theory, the frequency of plasma wave oscillations $f$ depends on the gate length $L_{\mathrm{g}}$, on the plasma wave velocity s, and on the drift velocity $v$ as $[7,10]$ :

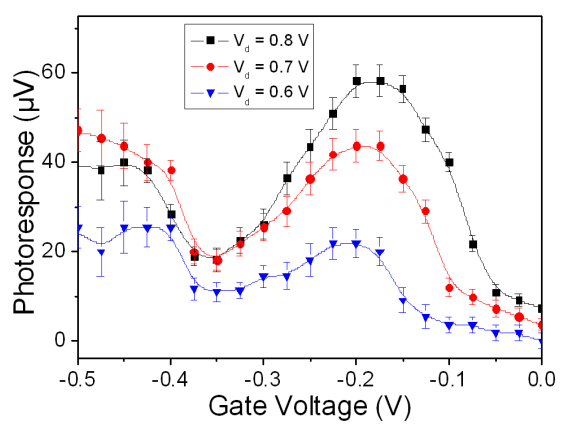

Fig. 2. Photoresponse as a function of the gate voltage for three different values of the applied drain voltage $(0.6 \mathrm{~V}, 0.7 \mathrm{~V}$, and $0.8 \mathrm{~V})$.

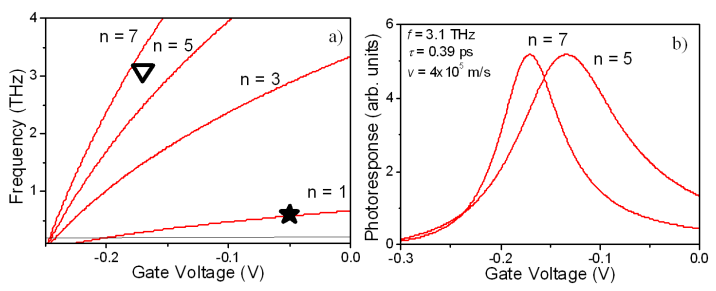

Fig. 3. (a) Frequency of the fundamental plasma mode and its harmonics up to the 7 th one as a function of the gate voltage using Eqs. (2) and (3). The triangle represents the position of the maximum of the resonant line at $3.1 \mathrm{THz}$ and the star corresponds to the resonance obtained at $600 \mathrm{GHz}$ in a different but similar transistor $[6,7]$. (b) Calculations using Eqs. (1) and (2) showing the signal corresponding to the 5 th and the 7 th harmonics of the fundamental plasma mode as a function of the gate voltage with $\tau=0.39 \mathrm{ps}$ and $v=4 \times 10^{5} \mathrm{~m} / \mathrm{s}$.

$$
f=\frac{s}{4 L_{\mathrm{g}}}\left(1-\frac{v^{2}}{s^{2}}\right)
$$

with

$$
s=\sqrt{\frac{e}{m^{*}} V_{0}},
$$

where $e$ is the electron charge and $m^{*}$ is the effective electron mass. Continuous lines in Fig. 3a are calculations using Eqs. (2) and (3) and describe the resonant fundamental frequency and its harmonics $(3,5,7)$ as a function of the gate voltage with a constant applied $V_{\mathrm{d}}$. The average electron drift velocity in the saturation regime is assumed to be $4 \times 10^{5} \mathrm{~m} / \mathrm{s}$.

The position of the line's maximum at $3.1 \mathrm{THz}$ is represented by a triangle in Fig. 3a and placed between the 5 th and the 7th calculated harmonics. The black star corresponds to the position of the resonant peak observed at $600 \mathrm{GHz}$ and already reported in the literature [6] in a similar device. In Fig. 3b the lines correspond to calculations using Eqs. (2) and (3) and showing the photoresponse signal for the 5 th and the 7 th harmonics of the fundamental plasma mode as a function of the gate voltage. One can see a good qualitative agreement between theory and the experimental curves of Fig. 2. Therefore, 
the observed peak can be attributed to the 5 th or the 7 th harmonics of plasma modes.

The quantitative interpretation based on these equations allows us getting only approximate description of experimental data. Indeed, simulations of the observed results require the knowledge of the non-homogeneous electron drift velocity along the channel, $v_{0}$, which is higher than the average electron saturation velocity. Indeed, Eq. (2) was derived assuming a constant velocity in the device channel and a constant momentum relaxation time. These assumptions are not valid in the transistor saturation region [11]. Moreover, the transistor channel region under the gate cannot be considered as separated from the other parts of the transistor. In a realistic structure the transistor consists not only of the gated part but also of the un-gated part that is covered by the cap layer [12]. In the non-equilibrium regime, the plasma frequency and the width of the resonant lines can be modified by the interaction of plasma oscillations in different parts of the channel. The resonant line is also wider than expected for this excitation frequency and this can be explained by previously described effect [13] and also by the propagation of oblique plasma modes $[14,15]$ into the transistors channel.

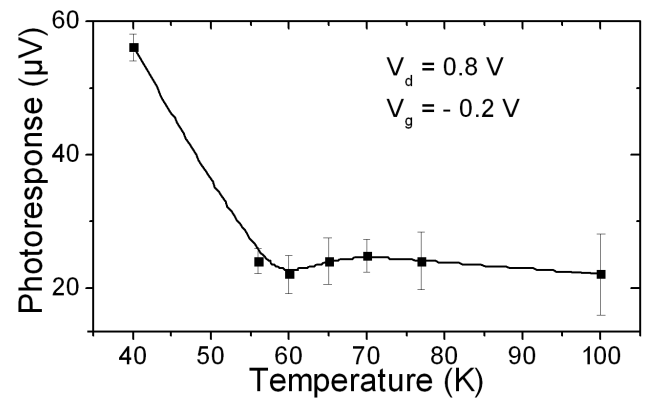

Fig. 4. Amplitude of the resonant line as a function of temperature for $V_{\mathrm{g}}=-0.2 \mathrm{~V}$ and $V_{\mathrm{d}}=0.8 \mathrm{~V}$. Signal is still measurable above $77 \mathrm{~K}$ even if it decreases and becomes noisy.

Finally, Fig. 4 shows the maximum of signal obtained for $V_{\mathrm{g}}=-0.2 \mathrm{~V}$ and $V_{\mathrm{d}}=0.8 \mathrm{~V}$ as a function of the QCL temperature from $40 \mathrm{~K}$ up to $100 \mathrm{~K}$. The signal decreases from $60 \mu \mathrm{V}$ down to approximately $20 \mu \mathrm{V}$ with a signal to noise ratio close to 3 . These results show that the $\mathrm{THz}$ system formed by the FET as a detector and the QCL as a source can be used even above liquid nitrogen temperatures.

\section{Conclusion}

In conclusion, we have shown the experimental evidence of the plasma wave resonant detection of pulsed $\mathrm{THz}$ radiation coming from a QCL at $3.1 \mathrm{THz}$ by GaAs FETs, both working above liquid nitrogen temperature. This solid-state system is compact and fast and therefore can be used for construction of $\mathrm{THz}$ space and time resolved imaging and spectroscopy systems.

\section{Acknowledgments}

This work was supported by CNRS and GDR-I project "Semiconductor sources and detectors of $\mathrm{THz}$ frequencies". We acknowledge the Region of LanguedocRoussillon through the "Terahertz Platform" project and the CNRS via the GIS Teralab. We want to thank Christian Lhenoret, Bertrand Mongellaz and Jean-Marie Peiris for technical support.

\section{References}

[1] B.S. Williams, Nature Photon. 1, 517 (2007), doi:10.1038/nphoton.2007.166.

[2] H. Luo, S.R. Laframboise, Z.R. Wasilewski, G.C. Aers, H.C. Liu, J.C. Cao, Appl. Phys. Lett. 90, 041112 (2007).

[3] D. Dragoman, M. Dragoman, Prog. Quant. Electron. 28, 1 (2004).

[4] M. Dyakonov, M.S. Shur, IEEE Trans. Electron. Dev. 43, 380 (1996).

[5] W. Knap, Y. Deng, S. Rumyantsev, M.S. Shur, Appl. Phys. Lett. 81, 4637 (2002); W. Knap, Y. Deng, S. Rumyantsev, J.-Q. Lü, M.S. Shur, C.A. Saylor, L.C. Brunel, Appl. Phys. Lett. 80, 3433 (2002).

[6] F. Teppe, D. Veksler, V.Yu. Kachorovski, A.P. Dmitriev, S. Rumyantsev, W. Knap, M.S. Shur, Appl. Phys. Lett. 87, 022102 (2005).

[7] F. Teppe, W. Knap, D. Veksler, M.S. Shur, A.P. Dmitriev, V.Yu. Kachorovskii, S. Rumyantsev, Appl. Phys. Lett. 87, 052107 (2005).

[8] F. Teppe, M. Orlov, A. El Fatimy, A. Tiberj, W. Knap, J. Torres, V. Gavrilenko, A. Shchepetov, Y. Roelens, S. Bollaert, Appl. Phys. Lett. 89, 222109 (2006).

[9] Fujitsu Microwave Semiconductor Databook (1999), Fujitsu Compound Semiconductor.

[10] D. Veksler, F. Teppe, AP. Dmitriev, V.Yu. Kachorovskii, W. Knap, M.S. Shur, Phys. Rev. B 73, 125328 (2006).

[11] H. Marinchio, G. Sabatini, C. Palermo, J. Pousset, J. Torres, L. Chusseau, L. Varani, P. Shiktorov, E. Starikov, V. Gružinskis, Appl. Phys. Lett. 94, 192109 (2009).

[12] P. Nouvel, H. Marinchio, J. Torres, C. Palermo, D. Gasquet, L. Chusseau, L. Varani, P. Shiktorov, E. Starikov, V. Gružinskis, J. Appl. Phys. 106, 013717 (2009).

[13] H. Marinchio, J.-F. Millithaler, C. Palermo, L. Varani, L. Reggiani, P. Shiktorov, E. Starikov, V. Gružinskis, Appl. Phys. Lett. 98, 203504 (2011).

[14] S. Boubanga-Tombet, F. Teppe, D. Coquillat, S. Nadar, N. Dyakonova, H. Videlier, W. Knap, A. Shchepetov, C. Gardès, Y. Roelens, S. Bollaert, D. Seliuta, R. Vadoklis, G. Valušis, 92, 212101 (2008).

[15] A. Shchepetov, C. Gardès, Y. Roelens, A. Cappy, S. Bollaert, S. Boubanga-Tombet, F. Teppe, D. Coquillat, S. Nadar, N. Dyakonova, H. Videlier, W. Knap, D. Seliuta, R. Vadoclis, G. Valušis, Appl. Phys. Lett. 92, 242105 (2008). 